\title{
Posttraumatic Arteriovenous Fistula of the Distal Posterior Tibial Artery as Cause of Delayed Wound Healing in an Unrecognized Arterial Injury
}

\author{
Peter Roth ${ }^{1}$ Christian Heiss ${ }^{2}$ Ahmed Koshty ${ }^{1}$ Bernd Niemann ${ }^{1}$ Andreas Boening ${ }^{1}$ \\ ${ }^{1}$ Herz-, Kinderherz-u Gefäßchirurgie, Universitätsklinikum Giessen, \\ Giessen, Germany \\ 2 Unfallchirurgie Giessen, Universitätsklinikum Giessen, Germany \\ Address for correspondence Dr. Peter Roth, MD, Herz-, Kinderherz-u \\ Gefäßchirurgie, UKGM, Campus Giessen, Rudolf-Buchheimstr. 7, Giessen \\ 35392, Germany (e-mail: Peter.Roth@chiru.med.uni-giessen.de).
}

Thorac Cardiovasc Surg Rep 2014;3:67-70.

\begin{abstract}
Keywords

- artery/arteries

- wound healing

- trauma

Background Cases of posttraumatic arteriovenous (AV) fistula of the distal lower limb are rarely reported. Time from trauma to diagnosis varies from hours to years.

Case Description This case report of a 51-year-old male patient shows very impressively the extensive consequences of an unrecognized traumatic AV fistula. The fistula developed here after a car accident led to a massive healing delay in a pilon fracture of the tibia and a wound, resulting in almost 1 year immobilization, 20 operations, and extensive pain drug therapy. After surgical revision of the fistula, bone and wound healing improved quickly.

Conclusions The possibility of an AV fistula should be considered in case of persistent swelling after high-energy trauma of an extremity. The therapy of choice is surgery and the long-term result of successful fistula ligature is excellent.
\end{abstract}

\section{Introduction}

A posttraumatic arteriovenous (AV) fistula of lower extremities is rare, but serious finding and detection is often difficult. Patients can be asymptomatic. If symptoms occur, they vary from edema to pain, even extending to heart failure due to high shunt volume. A thrill and bruit accompanying the AV shunt is characteristic, however, not always detectable. Many of the symptoms are reversible with surgery. We hereby present a case of posttraumatic AV fistula of the distal posterior tibial artery which persisted almost 1 year until recognition.

\section{Case Report}

A 51-year-old man was referred to our university hospital with a chronic pain in the swelling of the right foot. The patient had sustained a car accident 1 year ago which had caused an open pilon fracture of the right lower extremity. The fracture was surgically treated with an external fixation. Although the fracture was dislocated, open repositions were performed three times and the pilon fracture was stabilized with plates. The fibula was also stabilized with a plate (-Fig. 1). Wound necrosis developed in the area of the right ankle and debridement was performed several times and treated with continuous vacuum therapy (VAC). Due to disorder in wound healing, a hemisoleus muscle flap was performed which, however, did not lead to a better wound healing and revision was conducted 10 days later. Another wound debridement was performed in the sequel, whereby the wound was covered with a suralis plastic and meshgrafts, which were performed twice. An adequate wound healing was achieved under this treatment. The patient was then discharged with a pseudarthrosis, a swelling in the right lower limb and chronic pain, which was treated medically. received

August 19, 2013

accepted

November 4, 2013

published online

December 18, 2013
Dol http://dx.doi.org/

10.1055/s-0033-1363167. ISSN 2194-7635. (c) 2014 Georg Thieme Verlag KG
Stuttgart . New York

License terms

(1) (1) $\Theta \circledast$ 


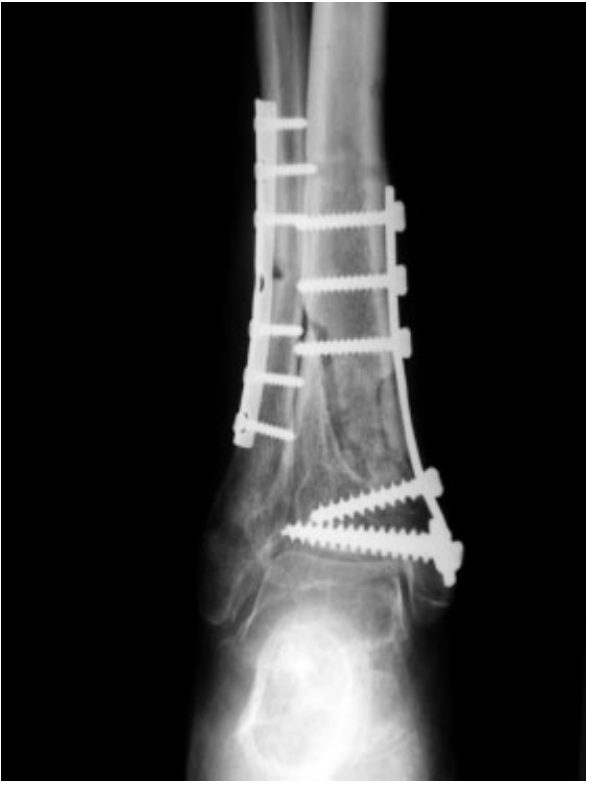

Fig. 1 The pilon fracture of the tibia stabilized with two plates.

A resection of the tibial pseudo-arthrosis was performed 3 months later. Nevertheless, the massive painful swelling persisted. Infection of the first toe necessitated an "Emmert Plastic," but the wound healing in the area was inhibited by the massive swelling. The patient was referred to the pain ambulance for the treatment of severe chronic pain.

One year after the car accident, the patient was referred to our hospital still immobile caused by pseudarthrosis of the distal tibia and fibula and was on continuous analgesic treatment because of the strong pain and extreme swelling in the right foot. The patient already had a history of 20 operations in three different hospitals. The right foot was extremely swollen ( - Fig. 2) with glossy skin and ulceration at the first toe. There was a superficial wound in the suralis flap plastic surrounding. Neither the distal pulses nor murmurs in the distal extremity were detectable.

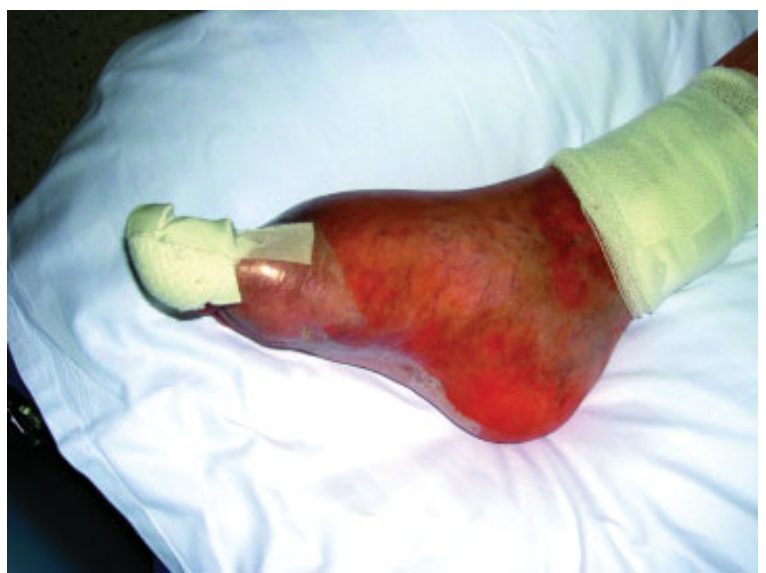

Fig. 2 Swelling of the right foot caused by an arteriovenous fistula of the posterior tibial artery with its concomitant veins.

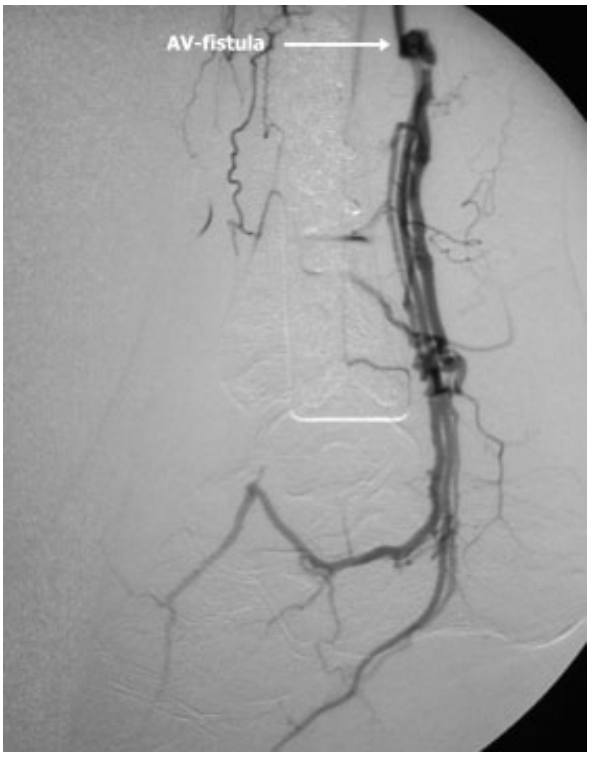

Fig. 3 Angiography of the arteriovenous (AV) fistula (arrow): There is reduced flow in the crural part of the artery visualized. The pertaining veins are occluded proximally and dilated peripherally due to blood drainage into the periphery veins.

A digital subtraction angiography was performed, showing an AV fistula of the distal posterior tibial artery including two of its concomitant veins. A saccular aneurysm had developed at the AV connection. The crural arteries were sparely visualized below the level of the fistula, while the veins were found to be enlarged ( - Fig. 3). Interestingly, the two veins involved were occluded proximally so that the arterial blood flow was drained not centrally but into the peripheral veins. An interventional therapy was excluded because of the one vessel blood supply of the lower leg and the risk of complete ischemia in case of an ineffective procedure.

We performed a surgical revision of the fistula. After localization of the fistula's exact position by X-ray in the operation theater, the posterior tibial artery was dissected. After preparation of the fistula and the pertaining veins, a small saccular aneurysm was divided and the two veins were sealed with $5 / 0$ suture. The segment of posterior tibial artery connected with the aneurysm was resected and an end-toend oblique reanastomosis was performed using 6/0 Prolene (Ethicon, Hamberg, Germany) suture.

The postoperative period was uneventful and wound healing was normal. The swelling decreased rapidly ( - Fig. 4) and the pain also eased off. The analgesic medication was reduced continuously. The size of the wound diminished quickly. The postoperative control angiography 14 days later showed patency of the posterior tibial artery without any persisting AV connection. The crural arteries demonstrated normal perfusion flow (-Fig. 5).

\section{Discussion}

Due to the increasing numbers of catheter interventions, AV fistulas are no longer a rarity. There is a huge amount of reports dealing with AV fistulas in the area of the groin and 


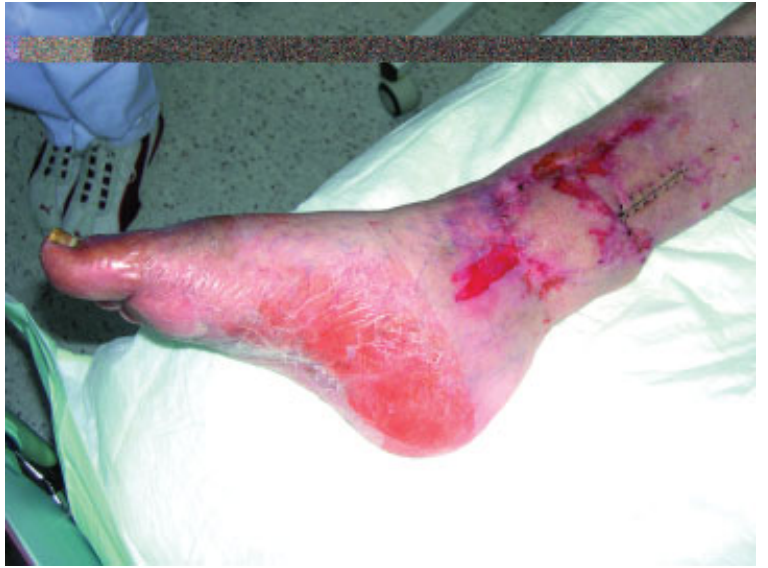

Fig. 4 The right lower leg 7 days after fistula ligature: Swelling had decreased and wound healing appears to be normal.

the vessels of the trunk. Traumatic AV fistulas are predominantly connected with bullet and stab wounds. ${ }^{1,2}$ On the contrary, traumatic AV fistulas in the distal extremities are rare. Diagnosis is often done with delay. ${ }^{3-5}$ This case report shows very impressively the extensive consequences of an unrecognized traumatic AV fistula. The fistula developed here after a car accident led to a massive healing delay in the tibia pilon fracture and the wound, resulting in almost 1 year

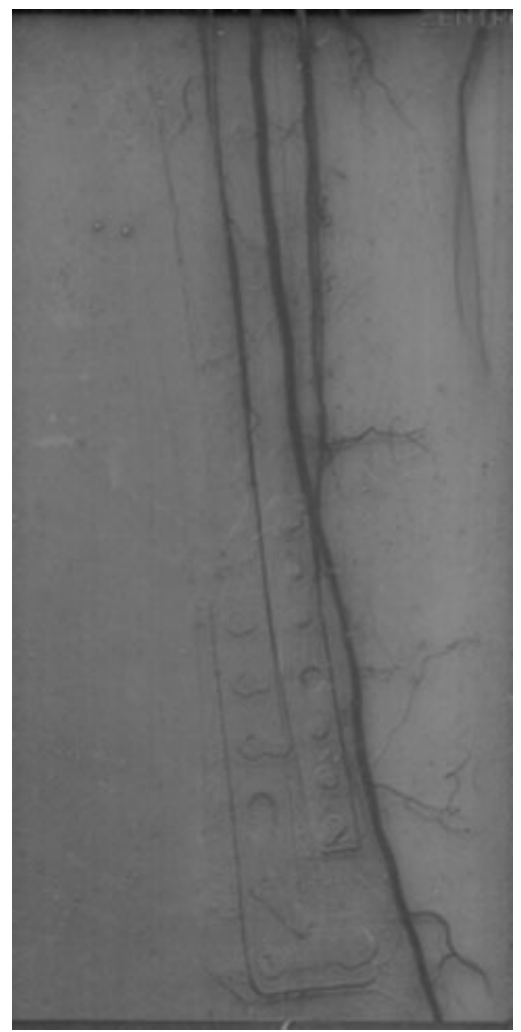

Fig. 5 Angiography of the right lower extremity 14 days after surgery: The posterior tibial artery appears normal without any residual fistula or stenosis. The erasement of the contrast media at two sites of the tibial artery is caused by hemostatic clips which were used subcutaneously. immobilization, 20 operations, and extensive pain drug therapy. During the long treatment, the patient was admitted to psychiatry because of depression and suicidal tendency caused by the sustained immobilization and unsuccessful therapy. Diagnosis of an AV fistula is often delayed because the main attention is given to treatment of the primary injuries. There is an array of typical symptoms, which unfortunately can either be partially or completely absent. Since swelling is common during trauma, it is not usually attributed to an AV fistula. In the reported case, there was also the exceptional finding of a central occlusion of the involved veins, leading to AV fistula drainage only to the peripheral veins, which contributed to a peripheral venous congestion with massive swelling. The ensued tissue pressure inhibited fracture healing and led to increased vulnerability of the tissue.

It is imperative to always treat a diagnosed AV fistula, since the ensuing shunt could lead to a volume load in the heart and even progress to cardiac insufficiency. ${ }^{6}$ Another feature of an AV fistula is vascular obstruction with organ ischemia and aneurysm formation. In many cases, interventional therapy (stent implantation, coiling, and thrombin injections) is possible within the region of the trunk. ${ }^{7,8}$ In the presented case, a vascular intervention was excluded because of occlusion of two of three vessels and the risk of complete ischemia of the foot. The surgical therapy entails a ligature of the vessels involved or exposure and ligature of the fistula and subsequently reconstructing the affected artery. Distally positioned AV fistulas, as in this case, are rarely treated interventionally because of the thin caliber of the vessels. Furthermore, because of the poor arterial collateralization, it is usually necessary to reconstruct the artery to maintain blood supply of the extremity. In the case of the patient described in this case report, this was performed by end-toend anastomosis of the posterior tibial artery.

The long-term clinical course after treating this AV fistula is impressive. The swelling of the lower leg receded quickly. Healing of the involved bone and skin improved rapidly with complete wound closure. After 4 years, there is still a certain constriction in movement in the upper subtalar joint. Circulation and skin condition are without pathological findings.

Traumatic AV fistulas in the distal extremities are rare, very often overlooked during acute trauma treatment and can have extensive consequences. The possibility of an AV fistula should be considered in case of persistent swelling after trauma of an extremity.

In the case presented here, diagnosis of the AV fistula was done by angiography, which was performed because of malperfusion of the foot. The most common method of detecting an AV fistula is duplex ultrasound examination. Duplex ultrasound is noninvasive and a direct identification of a fistula is possible in most cases. The color duplex imaging shows a bruit and/or thrill in the area of the fistula. In addition, the spectral Doppler analysis of the effected artery shows an elevated systolic and diastolic velocity. The pulsatility of the artery distal to the fistula is often normal. The effected vein shows an arterialized flow proximal to the fistula and a lack of respiratory phasicity. In the case of an 
70 Posttraumatic Arteriovenous Fistula of the Distal Posterior Tibial Artery Roth et al.

ongoing posttraumatic swelling of an extremity, an angiologic evaluation is recommended and a duplex ultrasound investigation should be performed. Angiography is also a safe and effective method of diagnosis and can be helpful in surgical planning.

The therapy of choice after diagnosis of an AV fistula in a distal extremity is surgery and the long-term result of successful fistula ligature is excellent.

\section{References}

1 Lerma R, Galego G, Lisbona C, Martorell A, Silveira P, Callejas JM. Arteriovenous fistulae of posttraumatic origin: apropos 7 cases [in Spanish]. Angiologia 1992;44(2):45-49

2 Davidovic L, Lotina S, Vojnovic B, et al. Post-traumatic AV fistulas and pseudoaneurysms. J Cardiovasc Surg (Torino) 1997;38(6): 645-651
3 Robbs JV, Carrim AA, Kadwa AM, Mars M. Traumatic arteriovenous fistula: experience with 202 patients. Br J Surg 1994;81(9): 1296-1299

4 Rostad H, Engedal H, Hall KV. Traumatic arteriovenous fistulas. A follow-up study. Angiology 1976;27(3):157-164

5 Touzard RC. A case of anterior tibial arteriovenous fistula after closed fracture of the leg [in French]. J Chir (Paris) 1975;109(5-6): 639-642

6 Ministro A, Costa T, Cunha e Sá D, Evangelista A, da Gama AD. High output heart failure due to an iatrogenic arteriovenous fistula after cardiac catheterization [in Portuguese]. Rev Port Cir Cardiotorac Vasc 2009;16(3):163-169

7 Ananthakrishnan G, DeNunzio M, Bungay P, Pollock G, Fishwick G, Bolia A. The occurrence of arterio-venous fistula during lower limb subintimal angioplasty: treatment and outcome. Eur J Vasc Endovasc Surg 2006;32(6):675-679

8 Peynircioglu B, Cil B. Amplatzer stuffing technique in the treatment of an iatrogenic mesenteric arteriovenous fistula. Cardiovasc Intervent Radiol 2009;32(6):1247-1251 